\title{
Fate of carbon during the formation of Earth's core
}

\author{
INGRID BLANCHARD ${ }^{1,2}$, ELEANOR JENNINGS ${ }^{3}$, IAN \\ FRANCHI $^{4}, \mathrm{XUCHAO} \mathrm{ZHAO}^{4}, \mathrm{SYLVAIN} \mathrm{PETITGIRARD}^{2}$, \\ NOBUYOSHI MIYAJIMA ${ }^{2}$, SETH A JACOBSON ${ }^{5}$ AND$$
\text { DAVID RUBIE }{ }^{2}
$$ \\ ${ }^{1}$ University of Potsdam \\ ${ }^{2}$ Universität Bayreuth \\ ${ }^{3}$ Birkbeck, University of London \\ ${ }^{4}$ Open University \\ ${ }^{5}$ Michigan State University \\ Presenting Author: blanchard@uni-potsdam.de
}

Carbon is an element of great importance on Earth, because it is intimately linked to the presence of life at the surface, and, as a light element, it may contribute to the density deficit of the Earth's iron-rich core. Carbon is strongly siderophile at low pressures and temperatures [1], hence it should be stored mainly in the Earth's core. Nevertheless, we still observe the existence of carbon at the Earth's surface, stored in crustal rocks, and in the mantle, as shown by the exhumation of diamonds. The presence of carbon in the crust and mantle could be the result of the arrival of carbon during late accretion after core formation ceased or because of a change in its metal-silicate partitioning behavior at the high pressures and temperatures of core formation ( $P>40 \mathrm{GPa}, T>3500 \mathrm{~K}$ ). We performed laser-heated diamond anvil cell experiments in order to quantify carbon partitioning behavior between liquid metal and silicate at the extreme conditions (49-71 GPa and 3600-4000 K) of Earth's core-mantle differentiation. We recovered our samples using the Focused Ion Beam technique and welded a $3 \mu \mathrm{m}$ thick slice of each sample onto a TEM grid. Major elements were analyzed by electron microprobe, whereas the concentrations of carbon in the silicate were analyzed by NanoSIMS. We thus have obtained metal-silicate partitioning results for carbon at $P T$ conditions relevant to planetary core formation. While $\mathrm{C}$ remains siderophile in all experiments, its partition coefficient is up to two orders of magnitude lower than in low PT experiments, as observed by [2]. We derive a new parameterization of the $P T$ dependence of the metal-silicate partitioning of carbon and apply this in a state-of-the-art model of planet formation and differentiation [3] that is based on astrophysical N-body accretion simulations. Results show that BSE carbon concentration increases starting at a very early stage of Earth's accretion and can reach estimated BSE values without requiring additional carbon delivery by late accretion events.

[1] Dasgupta et al., 2013. GCA 102, 191-212

[2] Fischer et al., 2020. PNAS 117, 8743-8749

[3] Rubie et al., 2015. Icarus 248, 89-108 